Published in final edited form as:

Ecol Lett. 2014 June ; 17(6): 717-726. doi:10.1111/ele.12276.

\title{
Natural soil microbes alter flowering phenology and the intensity of selection on flowering time in a wild Arabidopsis relative
}

\author{
Maggie R. Wagner, \\ Duke University Program in Genetics and Genomics, Department of Biology, Box 90338 Duke \\ University, Durham, NC 27708, USA
}

\section{Derek S. Lundberg,}

Department of Biology, Howard Hughes Medical Institute, Curriculum in Genetics and Molecular Biology, Carolina Center for Genome Sciences University of North Carolina, Chapel Hill, NC 27599, USA

\author{
Devin Coleman-Derr, \\ Joint Genome Institute, Walnut Creek, CA 94598, USA \\ Susannah G. Tringe, \\ Joint Genome Institute, Walnut Creek, CA 94598, USA
}

Jeffery L. Dangl, and Department of Biology, Howard Hughes Medical Institute, Curriculum in Genetics and Molecular Biology, Department of Microbiology and Immunology, Carolina Center for Genome Sciences University of North Carolina, Chapel Hill, NC 27599, USA

\section{Thomas Mitchell-Olds}

Institute for Genome Sciences \& Policy, Department of Biology, Box 90338 Duke University, Durham, NC 27708, USA

\begin{abstract}
Plant phenology is known to depend on many different environmental variables, but soil microbial communities have rarely been acknowledged as possible drivers of flowering time. Here we tested separately the effects of four naturally occurring soil microbiomes and their constituent soil chemistries on flowering phenology and reproductive fitness of Boechera stricta, a wild relative of Arabidopsis. Flowering time was sensitive to both microbes and the abiotic properties of different soils; varying soil microbiota also altered patterns of selection on flowering time. Thus, soil
\end{abstract}

CORRESPONDING AUTHOR: Maggie R. Wagner maggie.r.wagner@gmail.com phone: 1-919-668-6590 fax: 1-919-660-7293 post: Duke University Dept. of Biology, Box 90338, Durham, NC, USA 27708.

derek.lundberg@gmail.com

colemanderr@gmail.com

sgtringe@lbl.gov

dangl@email.unc.edu

tmo1@duke.edu

STATEMENT OF AUTHORSHIP: MRW collected soils, designed and executed the experiment, analyzed data, wrote permutation test scripts, and wrote the manuscript. MRW and TMO designed statistical models. DSL extracted and prepared DNA for sequencing and managed OTU pipeline. SGT and DC coordinated DNA amplification and sequencing. MRW, DSL, JLD, and TMO contributed to manuscript revisions.

CONFLICT OF INTEREST: The authors declare no conflict of interest. 
microbes potentially contribute to phenotypic plasticity of flowering time and to differential selection observed between habitats. We also describe a method to dissect the microbiome into single axes of variation that can help identify candidate organisms whose abundance in soil correlates with flowering time. This approach is broadly applicable to search for microbial community members that alter biological characteristics of interest.

\section{Keywords}

Phenology; plasticity; microbiome; soil ecology; selective agents; flowering time; selection; lifehistory; plant-microbe interactions

\section{INTRODUCTION}

Dissecting complex environments into biotic and abiotic components for individual study may lead to surprising and novel discoveries about how organisms respond phenotypically and evolutionarily to their habitats. This is because the net effect of a given environment is the accumulation of effects due to all relevant components of the ecosystem. Selection on floral characteristics, for instance, is mediated by many agents including pollinators, herbivores, temperature, and water availability (Strauss \& Whittall 2006). Thus, the complexity of natural habitats can preclude identification of the precise stimulus for phenotypic change or natural selection in the field (Anderson et al. 2013). By isolating simpler components of complex habitats, we can test whether each affects the phenotype expressed by a given genotype, the adaptive value of that phenotype, or both. These two effects of environment-phenotypic plasticity (Bradshaw 1965) and differential selection (Wade \& Kalisz 1990) — are crucial to understanding trait evolution and fitness both in a historical context and in the context of a changing planet. Despite their importance, most ecological drivers of trait expression and natural selection are unknown (MacColl 2011). Because environmental effects are best understood as responses of one trait to a specific stimulus (Bradshaw 1965), disentangling the precise ecological interactions that cause plasticity and differential selection in nature is an important goal (MacColl 2011).

For plants, soil is a key component of the complex natural habitat. Soils contain intricate patterns of chemical, physical, and microbial variation that are linked on continental (Fierer \& Jackson 2006) and centimeter scales (Berg \& Smalla 2009; Ettema \& Wardle 2002).

Feedbacks between above-ground plant communities, below-ground microbial communities, and nutrient availability are common (Berg \& Smalla 2009; Van der Heijden et al. 2008;

Ettema \& Wardle 2002). At the level of populations and individual plants, soil microbes can affect plant growth (van der Heijden et al. 2008; Rodriguez \& Falga 1999), resistance to infection (Berendsen et al. 2012) and aboveground herbivory (Hol et al. 2010). Additionally, microbes can mediate adaptation to novel environments (Lau \& Lennon 2012). Subsets of the soil microbiome interact with plants by colonizing aerial plant tissues (Vorholt 2012) or the rhizosphere or root (Bulgarelli et al. 2013; Bulgarelli et al. 2012; Lundberg et al. 2012). Thus, soil chemistry, plant biology, and microbial ecology are intricately linked (Berg \& Smalla 2009; Ettema \& Wardle 2002) and soils are comprised of many potential biotic and abiotic selective agents. 
In this study, we investigated the role of soil as a driver of plasticity and as an agent of selection on flowering time, an important ecological trait for plants and their communities (Forrest \& Miller-Rushing 2010). Flowering phenology has a strong genetic component (Brachi et al. 2010) but also responds to stimuli including temperature (Aikawa et al. 2011), water availability (Crimmins et al. 2013; Borchert et al. 2004), pathogen infection (Korves $\&$ Bergelson 2003), and herbivory (Brys et al. 2011). While soil chemistry is known to affect flowering time (Ryser \& Sauder 2006; Brun et al. 2003; Stanton et al. 2000; Pigliucci et al. 1995), soil microbial communities have rarely been acknowledged as possible drivers of reproductive timing in plants. Furthermore, previous studies that explored the relationship between soil microbiome, flowering, and selection used domesticated plants, artificial microbial communities, and/or biota from heavily disturbed soils (Lau \& Lennon 2012; Lau \& Lennon 2011; Batten et al. 2007). Although these experiments provide evidence that soil microbes change plant reproductive timing and selection pressures, they allow us to draw only limited conclusions about the evolutionary importance of this process.

Here, we asked whether (1) flowering phenology is plastic in response to different soil microbiomes, and (2) soil microbial communities alter the intensity of selection on flowering phenology. We further asked whether (3) relative abundance of specific members of the microbiome predict the observed effects of microbial treatments on flowering time. To test these hypotheses, we grew gnotobiotic (i.e. germinated in sterile conditions) seedlings of the non-mycorrhizal wild mustard Boechera stricta (Graham) Al-Shehbaz in sterilized potting soil inoculated with microbial communities extracted from soils of four undisturbed natural habitats. To enable comparison of biotic and abiotic soil variables, we also grew seedlings in field soils collected from the same habitats, which were sterilized to eliminate their natural microbiomes but retained their chemical and physical differences. For each individual plant, we recorded the day of first flowering, height, number of leaves, and number of fruits. We quantified selection on phenology as the linear relationship between flowering time and fruit production.

Rather than manipulating microbial communities, we used presumably intact communities extracted from soils collected from undisturbed field sites near wild B. stricta populations. Our experiment included 48 natural, inbred accessions that represent the breadth of genetic diversity harbored by $B$. stricta in the study region (central Idaho, USA). Furthermore, we focused on a phenotype (flowering time) that is under selection in this species in a nearby field site (Anderson et al. 2011). To our knowledge, no other study has tested whether naturally occurring soil biotas from multiple undisturbed habitats affect flowering phenology and selection on flowering time, nor has any study of the relationship between soil microbes and phenology explicitly accounted for genetic variation among undomesticated plant populations.

\section{MATERIAL AND METHODS}

In summary, we sterilized potting soil to kill the resident microbiome, and then inoculated subsets of the sterilized potting soil with microbial communities extracted from soils from four different field sites. To create four additional treatments that captured abiotic soil variation, we sterilized the same four field-collected soils. 
Sterilized potting soil was saturated with inoculum and sterilized field soils were saturated with buffer (see below) for 10 days. Then, 48 accessions of Boechera stricta were transplanted as gnotobiotic seedlings into all treatments (48 genotypes $\times 8$ treatments $\times 4$ replicates $=1,536$ individuals). All pots were randomized into blocks of 200 and maintained under controlled greenhouse conditions throughout the experiment, except for 7 weeks of $4^{\circ} \mathrm{C}$ vernalization in a growth chamber. We measured plant height and leaf number on the date of first flowering. Here we focus on phenology and fecundity of the $51 \%$ of plants that flowered successfully. Among those plants that flowered, the experimental design exhibited only modest imbalance, with substantial sample sizes remaining in every subspecies $\times$ treatment cell (mean $\mathrm{N}=54.6$, median $\mathrm{N}=56.5$ ). Factors influencing probability of flowering and other fitness components are beyond the scope of this paper; we found no evidence that any experimental treatments affected flowering probability of surviving plants $\left(\chi^{2}=8.86\right.$, $\mathrm{df}_{\text {num }}=7, P=0.26$ ). Reproductive fitness was estimated as the number of fruits on each individual at 33 weeks of age; in B. stricta, fruit set is strongly and positively correlated with seed production in the greenhouse $\left(\mathrm{R}^{2}=0.72, P<0.0001, \mathrm{~N}=103\right.$, A. Manzaneda 2008, unpublished).

\section{Soil and seed collections}

Soils were collected from four natural Boechera stricta habitats (described in Appendix S1 in Supporting Information) in central Idaho, USA, separated by $\sim 26$ to $\sim 92 \mathrm{~km}$ and differing in elevation, temperature, water availability, density and diversity of vegetation, and many soil properties (Wagner, unpublished). Collection locations were named 'Jackass Meadow' (abbreviated 'JAM'), 'Mahogany Valley' ('MAH'), 'Parker Meadow' ('PAR'), and 'Silver Creek' ('SIL'). These remote sites have little history of disturbance by humans, are home to endogenous $B$. stricta populations, and function well as common gardens for B. stricta field experiments (Rushworth et al. 2011). Therefore, they are legitimate potential habitats that $B$. stricta likely encountered during its evolutionary history in this region. Each soil collection (August 2012) comprised five sub-samples from the four corners and approximate center of a $\sim 150 \mathrm{~m}^{2}$ area, at a depth of $\sim 10$ to $30 \mathrm{~cm}$. Subsamples were combined and mixed thoroughly, sieved through $\sim 1.25 \mathrm{~cm}$ wire mesh to remove rocks and coarse detritus, shipped to Duke University, and stored in plastic bags at $4{ }^{\circ} \mathrm{C}$ for $\sim 3$ months until use. We also collected seven $\sim 1 \mathrm{~mL}$ soil vouchers from each site for microbial community analysis: three in August 2011, and at all sites except PAR, four in August 2012. Vouchers were frozen at $-20^{\circ} \mathrm{C}$ until DNA extraction in late 2012. B. stricta seeds were collected from 48 natural populations, including four from the soil collection sites. Their sites of origin span over 1,000 $\mathrm{m}$ in elevation (Table S1) and are separated by between $\sim 1 \mathrm{~km}$ and $\sim 350 \mathrm{~km}$, with the exception of the "SAD12" genotype from Colorado. Because $B$. stricta is naturally inbred and exhibits high $F_{\text {IS }}$ (i.e. individuals are largely homozygous) and $F_{\mathrm{ST}}$ (i.e. low genetic variation within populations and substantial divergence among populations; Song et al. 2006), each population was represented by a distinct genotype. This diverse collection of genotypes included 24 from each of the ecologically divergent "east" and "west" subspecies (Lee \& Mitchell-Olds 2013). We used seeds from a single mother descended from the original field-collected accession, self-pollinated in the greenhouse for at least one generation, to minimize maternal effects; i.e. individuals within a genotype were self-full sibs (as in Anderson et al. 2011). 


\section{Microbial treatments in sterilized potting soil}

To create four soils that were identical except for their microbial communities, we extracted microbes from field soils into sterile buffer and soaked sterilized potting soil in the resulting suspensions. We prepared inocula from $75 \mathrm{~g}$ subsamples of each field-collected soil stirred into $1 \mathrm{~L}$ of $2.5 \mathrm{mM}$ MES monohydrate (Sigma Aldrich, St. Louis, MO, USA) in sterile $\mathrm{diH}_{2} \mathrm{O}$ ( $\mathrm{pH}$ adjusted to 5.7 with $\mathrm{KOH}$ ). After settling for $30 \mathrm{~min}$ the suspensions were vacuum filtered (11 $\mu \mathrm{m}$ pore size) to remove particulates. Filtrates were centrifuged $30 \mathrm{~min}$ at $3,000 \times \mathrm{G}$ at room temperature to pellet microorganisms. To remove dissolved nutrients, we discarded the supernatants and re-suspended the microbe-enriched pellets in $1 \mathrm{~L}$ sterile $2.5 \mathrm{mM}$ MES. This process mostly eliminated variation in chemical properties that differentiate the field soils (Fig. S1, Table S2). Each rack of 200 pots was bottom-saturated with $400 \mathrm{~mL}$ of one of the microbial suspensions, $6 \mathrm{~g}$ 20-10-20 fertilizer, and sterile $\mathrm{diH}_{2} \mathrm{O}$ for a total treatment volume of $4 \mathrm{~L}$. The fertilizer was added to encourage seedling survival and to counteract possible soil impoverishment due to autoclaving (Berns et al 2008). An additional $1 \mathrm{~mL}$ of undiluted microbial suspension was pipetted into each pot. Treatments derived by this process are termed "biotic" or "microbial treatments" throughout. It is possible that the filtration and re-colonization processes somewhat altered community structures; however, the differences between our experimental inocula - and their effects on the plants—originate from corresponding differences between real Boechera habitats.

\section{Sterilized field soil treatments}

We sterilized soils from four natural habitats to create growth substrates with different physical and chemical properties, but without their natural microbiomes. After sub-sampling to extract microbial communities (see above), we sterilized the four field-collected soils via autoclaving (Appendix S2). These soils were loosely packed into clean pots and bottomsaturated with $400 \mathrm{~mL}$ sterile $2.5 \mathrm{mM}$ MES, $6 \mathrm{~g}$ 20-10-20 fertilizer, and sterile $\mathrm{diH}_{2} \mathrm{O}$ to bring the treatment volume to $4 \mathrm{~L}$. An additional $1 \mathrm{~mL}$ sterile $2.5 \mathrm{mM}$ MES was pipetted into each pot. Treatments derived by this process are termed "abiotic" or "sterilized field soils". Although it is likely that autoclaving these soils changed their fertility, they appear to have retained at least some of their natural chemical variation (Fig. S1).

\section{Plant care and trait measurement}

Surface-sterilized seeds of 48 genotypes (Table S1) were stratified on autoclaved filter paper at $4{ }^{\circ} \mathrm{C}$ in the dark for two weeks, then placed in a growth chamber to germinate for one week (conditions in Appendix S2). Four germinated seedlings per genotype were transplanted into each of the eight experimental soils described above, one seedling per pot. Eight pots per treatment were left unplanted as controls. All pots were immediately rearranged into randomized blocks and maintained in controlled greenhouse conditions (Appendix S2) for the duration of the experiment. Plants were top-watered as needed with $\mathrm{RO}$ water, and received an additional $4 \mathrm{~mL}$ 20-10-20 fertilizer (dissolved in sterile $\mathrm{diH}_{2} \mathrm{O}$ ) via pipet when one month old. Two-month-old plants were transferred to a $4{ }^{\circ} \mathrm{C}$ vernalization treatment, where they remained for seven weeks.

After vernalization, plants were returned to the greenhouse, checked three times weekly for flowers and allowed to set fruit. Flowering was defined as sufficient separation of the corolla 
such that four distinct petals could be identified. The number of days between end of vernalization and first flowering is termed interchangeably "flowering time" and "flowering phenology" throughout. On the day of first flower for each plant we measured the individual's height (defined as the length of green tissue up to the apical meristem) and number of leaves. The last census was done eight weeks post-vernalization; the 749 plants that had not flowered by this date were excluded from all future analyses. The experiment ended two months after the final flowering census, when almost all fruits had matured and dehisced. These cutoffs for flowering time and fruit production are realistic given the short growing season observed in the field. At this time we counted the number of fruits produced by each individual.

\section{Soil bacterial analyses}

Due to current methodological limitations, in this study we focus on the prokaryotic component of the soil microbiome (i.e. bacteria and archaea). We extracted DNA from fieldcollected soil vouchers using the MoBio ${ }^{\mathrm{TM}}$ PowerSoil DNA Isolation Kit (MoBio Laboratories, Carlsbad, CA, USA) and amplified variable region 4 of the bacterial $16 \mathrm{~S}$ rRNA gene using established primer pairs 515F and 806R and PNA PCR clamps to reduce plastid and mitochondrial contamination (Lundberg et al. 2013). Paired-end $2 \times 250 \mathrm{bp}$ sequencing of barcoded amplicons was performed on a MiSeq machine running v2 chemistry (Illumina Inc., San Diego, CA, USA) at the Joint Genome Institute (Walnut Creek, CA, USA). The primer sequences were trimmed from the paired-end sequences, which were then overlapped and merged using FLASH (Magoč \& Salzberg 2011). Merged sequences were grouped into operational taxonomic units (OTUs) based on 97\% sequence identity, and chimeric sequences were removed, using the UPARSE pipeline (Edgar 2013). Taxonomies were assigned as in Lundberg et al. (2012). Unclassifiable OTUs at the kingdom level, OTUs matching Viridiplantae, mitochondrial, or plastid sequences were excluded by using BLAST to compare them to a custom database of contaminant sequences (Lundberg et al. 2013). Unclassifiable OTUs at the kingdom level and rare or nonreproducible OTUs were also excluded as in Lundberg et al. (2012), resulting in 7,844 OTUs. To control for unequal sequencing effort, we normalized data by rarefaction to 40,000 reads/sample using QIIME-1.7.0 (Fig. S2; Caporaso et al. 2010). Diversity analyses were performed after correcting data for $16 \mathrm{~S}$ gene copy number variation using scripts provided in Kembel et al. (2012); OTUs without taxonomic information were assigned the mean copy number (2.805725). Linear regressions were performed before this correction but the resulting parameter estimates were adjusted as necessary.

\section{Statistical analysis}

To test the hypothesis that soil properties affect flowering time, we used restricted maximum likelihood (REML) linear mixed models with treatment, subspecies, and treatment $x$ subspecies as fixed factors; block, genotype nested within subspecies, and treatment $x$ genotype as random factors; and elongation rate ( $\mathrm{mm} /$ day), height at first flowering $(\mathrm{mm})$, and leaves per mm stem as covariates (justification in Appendix S2). To test for treatment effects on overall plant size we used MANOVA with treatment, subspecies, and treatment $x$ subspecies as fixed factors. We analyzed the parallel "biotic" and "abiotic" experiments separately, i.e. one model tested only for effects of soil microbiomes on flowering time, and 
another identical model tested only for effects of physical soil differences; we did not directly compare the effects of these two types of soil variation. These models were run using JMP® Pro version 10.0.0 (SAS Institute, Cary, NC, USA). Statistical significance of random effects was determined by REML likelihood ratio test and results were graphed using ggplot2 (Wickham 2009) in $\mathbf{R}$ version 3.0.2 (R Core Team 2013).

To test the hypothesis that soil properties alter selection on flowering time, we used a REML linear mixed model with the same terms as above, plus flowering time (days after vernalization) and flowering time $\times$ treatment as additional fixed effects. The response variable was number of fruits. Thus, the flowering time term describes the change in fecundity attributed to a change in flowering time, i.e. selection. We analyzed the parallel "biotic" and "abiotic" experiments separately as above. We performed these models both with and without including elongation rate, height at first flowering, and leaves per $\mathrm{mm}$ stem as covariates; the former model describes the selection gradient on flowering time (i.e. direct selection on flowering time independent of selection on covariates), and the latter model describes the selection differential (i.e. total selection on flowering time, including indirect effects of selection on covariates). Introducing a quadratic term did not improve fit, so our model considers only linear effects (i.e. directional selection). Sample sizes were slightly smaller than for the flowering time models because 17 individuals were accidentally discarded after flowering. Main models were performed in JMP; selection differentials and selection gradients were calculated in $\mathbf{R}$.

For some models, non-uniformly distributed residuals might have influenced judgments about significance. This heteroscedasticity resisted data transformation and resolved only when covariates were removed. In general, results with and without covariates were similar, suggesting that significance was robust to heteroscedasticity in the standard model. For the sake of caution, for all of our major results, we performed permutation tests (which do not assume uniform residuals) to verify the results of the standard ANOVA.

Rarefied microbial communities were analyzed in the $\mathbf{R}$ package 'vegan' (Oksanen et al. 2013). Principal coordinates (PCo) of Bray-Curtis pairwise dissimilarities were identified using the vegan function 'capscale'. Similarity of samples within vs. among sites was tested using the non-parametric permutation test ADONIS with 9,999 permutations constrained by collection year. To ask which components of microbial communities affect flowering phenology and selection, we regressed mean flowering time in each biotic Treatment (using residuals from the linear models described above in Table 1a, excluding terms with the "Treatment" factor) onto the mean PCo score from the corresponding Site. To ask which OTUs underlie the observed phenotypic effect, we identified the ten OTUs most highly correlated with the PCo axes and regressed the same flowering time residuals on the OTUs' mean abundances at each site. We used the Wilcoxon rank-sum test to compare relative abundances of common taxa between groups of samples associated with extreme phenotypes. P-values were adjusted using Benjamini-Hochberg false discovery rate. Our method of searching for microbial community members that underlie our phenotype of interest is described in more detail in Appendix S3. 


\section{RESULTS}

\section{Microbial communities and physical soil types each alter mean flowering time}

Soil microbiota altered mean flowering time across all genotypes (Fig. 1a; Table 1a; $F_{3,87}=4.242, P=0.008$; permutational ANOVA $P=0.005$ ). In particular, the MAH microbiome delayed flowering by 2.2 days on average (or 1.5 days when controlling for plant size and growth rate; Table S3). This delay translates to a $9 \%$ fitness decrease based on the selection differential measured at a similar field site (Anderson et al. 2011). Although western genotypes appear more sensitive to microbes than eastern genotypes (Fig. 1a,c), the subspecies difference in microbiome-driven plasticity of flowering time was not significant (Treatment $\times$ subspecies: $F_{3,88}=2.346, P=0.078$ ). Genotype explained $34.5 \%$ of the variance in flowering time, indicating substantial among-population genetic variation for flowering phenology across all microbiomes $\left(\chi^{2}=30.08, \mathrm{df}_{\text {num }}=2, P<0.0001\right)$. In contrast, genotype $\times$ treatment explained no variation, suggesting a lack of genetic diversity for flowering time plasticity in response to soil microbiota. Excluding growth-related covariates from the model did not qualitatively change the results (Table S4a). Neither soil microbiome (MANOVA Wilks' $\lambda=0.982, \mathrm{~F}_{9,1073}=0.90, P=0.53$ ) nor subspecies $\times$ soil microbiome (MANOVA Wilks' $\lambda=0.976, \mathrm{~F}_{9,1073}=1.21, P=0.28$ ) affected overall plant size.

Differences between sterilized field soils also affected flowering phenology (Figs. 1b,1d; Table $\left.1 \mathrm{~b} ; F_{3,96}=6.586, P=0.0004\right)$. PAR soil had the most extreme effect, delaying flowering by 2.8 days after controlling for effects on growth. Again, the subspecies did not differ in their flowering time response to soil type (Fig. $1 \mathrm{~d} ; F_{3,91}=0.206, P=0.8919$ ). Genotype explained only $5.7 \%$ of the variance in flowering time $\left(\chi^{2}=0.864, \mathrm{df}=2, P>0.05\right)$ and genotype $\times$ treatment explained $4.72 \%\left(\chi^{2}=0.160, \mathrm{df}=6, P>0.05\right)$, indicating scant genetic variation for flowering time plasticity in response to abiotic soil variation. However, genetic effects were more pronounced when growth covariates were excluded from the model (Table S4b).

\section{Microbial community, but not soil chemistry, alters selection on flowering time}

Selection on flowering time depended on soil microbiome. This result held both for selection gradients (independent of selection on correlated traits; Fig. 2c, Table 2a; $F_{3,246}=3.489, P=0.016$; permutational ANOVA $P=0.024$ ) and selection differentials (net selection on flowering time, including indirect effects of selection on covarying traits; Fig. 2a, Table S5a). The most extreme change was between the PAR and SIL soil biotas (Fig. 2a), with selection differentials of +0.034 and -0.043 fruits/day (or $+1.4 \%$ and $-1.8 \%$ fruits/ day), respectively. The magnitude of this difference in selection is 1.2 times the selection differential measured in a nearby field site (Anderson et al. 2011). We detected no directional selection on flowering time when averaged across all treatments $\left(F_{1,403}=0.009\right.$, $P=0.924)$, suggesting that treatment differences in selection intensity cancelled each other out. On average, western genotypes produced 0.75 more fruits than eastern-a $27 \%$ increase $\left(F_{1,69}=7.351, P=0.008\right.$; Table $\mathrm{S} 3$ ). In addition, genetic differences between populations within subspecies contributed $20.4 \%$ of the variance in fecundity $\left(\chi^{2}=13.79, \mathrm{df}=2, P<0.01\right)$. However, the lack of subspecies $\times$ treatment and genotype $\times$ treatment interactions shows that genetic effects on performance were not microbiota-dependent. Finally, fitness was 
consistent across microbial treatments $\left(F_{3,86}=2.123, P=0.103\right)$, indicating no net effect of different microbiomes on fecundity (Tables 2a, S3).

In contrast, we found no evidence that different sterilized field soils affect flowering time selection differentials (Fig. $2 \mathrm{~b} ; F_{3,301}=0.809, P=0.17$ ) or gradients (Fig. $2 \mathrm{~d} ; F_{3,305}=1.552$, $P=0.20)$. However, these treatments affected overall fecundity $\left(F_{3,110}=5.849, P=0.001\right.$; Table S3), indicating strong differences in soil quality. Western genotypes produced $69 \%$ more fruits than eastern genotypes on average $\left(F_{1,64}=16.13, P=0.0002\right)$, but this advantage was consistent across all soils (Table S3). We detected no net selection gradient on flowering time across all field soils $\left(F_{1,308}=0.376, P=0.540\right)$; however, we did find a significant selection differential on flowering time (-0.036 or - $1.4 \%$ fruits/day; $F_{1,313}=6.473, P=0.011$; Table S5).

\section{Separation of microbiome components reveals finer details of selection and phenology control}

Sampled soil microbiomes differed between sites within year $\left(R^{2}{ }_{\text {ADONIS }}=0.29, P=0.003\right)$ but not between years within site $\left(R^{2}\right.$ ADONIS $\left.=0.03, P=0.56\right)$. These results are supported by principal coordinates (PCo) analysis, in which samples clustered mainly by site (Fig. 3a). The first three PCo cumulatively explained $69.8 \%$ of prokaryotic community variation (Fig. S3). Because the MAH and JAM soils, which had the slowest and fastest flowering times respectively in our experiment, were separated primarily on the PCo2 axis (Fig. 3a), PCo2 became our candidate microbiome component to test for effects on flowering time. Because eastern genotypes appeared insensitive to microbes (Fig. 1c), we used flowering time residuals of western genotypes as the response variable. We did not find a significant relationship between mean flowering time in each treatment and the mean $\mathrm{PCo} 2$ score of soil samples from the corresponding site (Fig. $3 \mathrm{~b} ; F_{1,2}=2.362 ; P=0.26$ ). Likewise, none of the individual OTUs with high PCo2 scores predicted flowering time (Table S6); for instance, abundance of OTU_96997 (Hyphomicrobiaceae) appeared to correlate with phenology but the relationship was nonsignificant after correction for multiple comparisons (Fig. 3c; $F_{1,2}=25.74 ; P=0.037 ; P_{\mathrm{FDR}}=0.38$ ). These negative results may be due to lack of power caused by our sampling scheme $(\mathrm{N}=4$; Appendix $\mathrm{S} 3)$.

The phyla Proteobacteria and Crenarchaeota were more abundant, and Acidobacteria were less abundant, in slow-flowering MAH compared to fast-flowering JAM soil communities (Table 3b). Within phyla, several families—including Koribacteraceae, Solibacteraceae, Opitutaceae, Verrucomicrobiaceae, Solirubrobacteraceae, and Mycobacteriaceae-differed in relative abundance between MAH and JAM soil communities (Fig. S4, Table S7). In addition, Verrucomicrobia and Gemmatimonadetes were enriched in the 5\% of OTUs with the highest loadings on PCo2 compared to the full natural communities (Table 3c), indicating that these phyla contribute disproportionately to the microbiome variation summarized by PCo2. These 'candidate taxa' are promising organisms for further study of microbial influences on flowering time. 


\section{DISCUSSION}

Our analysis of native plant genotypes and soil microbial communities from four undisturbed environments suggests that soil microbiomes contribute to the ecology and evolution of flowering phenology in Boechera stricta. First, we showed that soil microbiota influenced phenotypic plasticity of flowering time. Second, soil microbiota altered the strength and direction of selection on flowering time. Finally, we showed how this type of experiment could be combined with quantitative descriptions of soil community composition to search for microbial species that affect important phenotypes. Our results show that experimental dissection of complex environments can reveal the ecological interactions shaping phenotypic expression and natural selection.

Our finding that both soil microbes and soil chemistry cause plasticity of flowering time agrees with previous reports, although to our knowledge only one other study has tested the effects of both biotic and abiotic components of the same soil. Lau \& Lennon (2012) found that Brassica rapa flowered faster in dry conditions, and that a soil microbial community with a history of drought stress accelerated flowering compared to the wet-adapted replicate of the same community. Other soil properties reported to delay flowering include heavy metal concentration (Ryser \& Sauder 2006; Brun et al. 2003), nutrient depletion (Stanton et al. 2000; Pigliucci et al. 1995), high salinity (Van Zandt \& Mopper 2002), and a history of invasive plant growth (Batten et al. 2007). Notably, some species' phenology may be more robust than others' in response to soil heterogeneity (Batten et al. 2007). In general, such phenotypic plasticity has important ecological and evolutionary consequences (Ghalambor et al. 2007; Richards et al. 2006; Miner et al. 2005; Thompson 1991; Bradshaw 1965). For B. stricta in particular, phenological plasticity affects the plants' ability to time reproduction for optimal seed set (Anderson et al. 2011). In fact, the exclusion of natural soil microbiomes from growth chamber replicates of that experiment might be partially responsible for the low genetic correlation of flowering time in the field and in the lab (Anderson et al. 2011). Interestingly, our data hint that West genotypes may be more sensitive to soil microbiome than East genotypes ( $P=0.078$; Fig. 1a,c; Table 1a), suggesting intraspecific genetic variation for microbe-induced flowering time plasticity. In contrast, the two subspecies show very similar sensitivities to abiotic soil properties (Fig. 1d), indicating that plasticity to these two stimuli may have different genetic bases.

Typically, selection analyses allow us to infer and compare the adaptive value of particular traits in particular environments, but do not tell us why differential selection exists. Here we identified the soil microbiome as an agent of selection on flowering time in B. stricta (Figs. $2 \mathrm{a}, \mathrm{c})$. The evolutionary relevance of this finding is best illustrated by comparison with Anderson et al. (2011), who measured a decrease in fecundity of 0.06 fruits/day to flowering ( $>4 \%$ of total mean fitness/day) in a typical B. stricta habitat. Our results suggest that all else remaining constant, a change in soil microbiome could increase or decrease that selection differential by up to 0.07 fruits/day, or $>120 \%$ (based on the magnitude of differential selection measured in the PAR and SIL soil communities; Fig. 2a). The effects of the soil microbiome on both flowering time and its adaptive value may prove especially important in the context of conservation and adaptation to global change, given that both 
microbial communities and plant phenology are sensitive to climate (Castro et al. 2009;

Cleland et al. 2007).

Although we are not the first to report that soil biotas affect flowering time (Lau \& Lennon 2012) or selection on flowering time (Lau \& Lennon 2011), our experiment adds to previous work in several unique ways. First, we used deep 16S rDNA sequencing methods to greatly enhance our resolution of the microbiome (Lundberg et al. 2013). Second, we described how this type of microbiome data can be combined with experimental phenotype data to determine which microbial taxa influence traits of interest. Third, including a diverse set of genotypes in the experiment allowed us to test for intraspecific genetic variation for flowering time plasticity in response to biotic and abiotic soil attributes, and link this ecological finding to the field of evolutionary biology. Finally, we are the first to use microbial communities from several undisturbed field sites to confirm that naturally occurring variation in soil microbes affects phenology and its adaptive value. We identified several taxa that are enriched or depleted in soils associated with fast flowering compared to slow flowering (Tables 3b, S4); these groups are promising targets for future study of the microbe-flowering time relationship.

As evolutionary biologists, a fundamental goal is to measure selection and trait expression in the field because the genotype-phenotype-fitness relationship is frequently contextdependent (Anderson et al. 2013). In particular, the true relationships between plants and microbial communities may depend on neighboring plants (Berg \& Smalla 2009) or on other soil properties (van der Heijden et al. 2008). For instance, Lau and Lennon (2012) found that the interaction between microbes and soil moisture synergistically affects fitness (but not phenology) in Brassica rapa. Factorial application of a wider sampling of microbiomes and environmental variables - or, eventually, direct manipulation of these variables in the field -would be especially informative for understanding the ecological mechanisms of plantmicrobe interactions such as the flowering time effect we observed in B. stricta.

Nonetheless, we demonstrate here that greenhouse experiments can reveal ecological interactions that may have been extremely difficult to detect directly in the field: all else remaining equal, natural differences in the soil microbiome can influence plant phenology and patterns of selection. This discovery required the isolation of soil microbiota from the larger, more complex natural habitat. Further environmental simplification-the reduction of the microbiome into principal coordinates and then specific OTUs-potentially could reveal even more details of the relationship between genotype, phenotype, and the microbial environment (Appendix S3). Although in this experiment we lacked power to fully utilize this method, it holds promise for future studies on the phenotypic effects of the environmental microbiome. This approach is generally applicable to search for microbial community members that alter biological characteristics of interest.

\section{Supplementary Material}

Refer to Web version on PubMed Central for supplementary material. 


\section{Acknowledgments}

MRW was supported by a Graduate Research Fellowship and a Doctoral Dissertation Improvement Grant from the National Science Foundation and NIH Training Grant 5T32 GM007754-32. TMO was supported by grant R01 GM086496 from the National Institutes of Health and EF-0723447 from the National Science Foundation. JLD was supported by NSF Microbial Systems Biology grant IOS-0958245 and DOE Plant Feedstocks grant DOESC0010423. DSL was supported by NIH Training Grant T32 GM07092-34. JLD is an Investigator of the Howard Hughes Medical Institute and support was provided by the Howard Hughes Medical Institute and the Gordon and Betty Moore Foundation (in part via Grant GBMF3030 to JLD). This work was also funded by the JGI Director's Discretionary Grand Challenge Program, and a JGI community Sequencing Project (SGT, JLD and TMO). Work conducted by the U.S. Department of Energy Joint Genome Institute is supported by the Office of Science of the U.S. Department of Energy under Contract No. DE-AC02-05CH11231. Many thanks to L.T. Burghardt and three anonymous reviewers for helpful comments and suggestions on earlier versions of the manuscript, and to J. Mays and the Duke greenhouse staff for watering assistance.

\section{REFERENCES}

1. Aikawa S, Kobayashi MJ, Satake A, Shimizu KK, Kudoh H. Robust control of the seasonal expression of the Arabidopsis FLC gene in a fluctuating environment. P. Natl. Acad. Sci. USA. 2010; 107:11632-11637.

2. Anderson JT, Lee CR, Mitchell-Olds T. Life-history QTLs and natural selection on flowering time in Boechera stricta, a perennial relative of Arabidopsis. Evolution. 2011; 65:771-787. [PubMed: 21083662]

3. Anderson JT, Wagner MR, Rushworth CA, Prasad KVSK, Mitchell-Olds T. The evolution of quantitative traits in complex environments. Heredity. 2014; 112:4-12. [PubMed: 23612691]

4. Batten KM, Scow KM, Espeland EK. Soil Microbial Community Associated with an Invasive Grass Differentially Impacts Native Plant Performance. Microbial Ecol. 2007; 55:220-228.

5. Berendsen RL, Pieterse CMJ, Bakker PAHM. The rhizosphere microbiome and plant health. Trends Plant Sci. 2012; 17:478-486. [PubMed: 22564542]

6. Berg G, Smalla K. Plant species and soil type cooperatively shape the structure and function of microbial communities in the rhizosphere. FEMS Microbiol. Ecol. 2009; 68:1-13. [PubMed: 19243436]

7. Berns AE, Philipp H, Narres H-D, Burauel P, Vereecken H, Tappe W. Effect of gamma-sterilization and autoclaving on soil organic matter structure as studied by solid state NMR, UV and fluorescence spectroscopy. Eur. J. Soil Sci. 2008; 59:540-550.

8. Borchert R, Meyer S, Felger R, Porter-Bolland L. Environmental control of flowering periodicity in Costa Rican and Mexican tropical dry forests. Glob. Ecol. Biogeog. 2004; 13:409-425.

9. Brachi B, Faure N, Horton M, Flahauw E, Vazquez A, Nordborg M, et al. Linkage and Association Mapping of Arabidopsis thaliana Flowering Time in Nature. PLoS Genet. 2010; 6:e1000940. [PubMed: 20463887]

10. Bradshaw AD. Evolutionary significance of phenotypic plasticity in plants. Adv. Genet. 1965; 13:115-155.

11. Brun LA, Le Corff J, Maillet J. Effects of elevated soil copper on phenology, growth and reproduction of five ruderal plant species. Environ. Pollut. 2003; 122:361-368. [PubMed: 12547525]

12. Brys R, Shefferson R, Jacquemyn H. Impact of herbivory on flowering behaviour and life history trade-offs in a polycarpic herb: a 10-year experiment. Oecologia. 2011; 166:293-303. [PubMed: 21120670]

13. Bulgarelli D, Rott M, Schlaeppi K, Ver Loren van Themaat E, Ahmadinejad N, Assenza F, et al. Revealing structure and assembly cues for Arabidopsis root-inhabiting bacterial microbiota. Nature. 2012; 488:91-95. [PubMed: 22859207]

14. Bulgarelli D, Schlaeppi K, Spaepen S, van Themaat EVL, Schulze-Lefert P. Structure and Functions of the Bacterial Microbiota of Plants. Annu. Rev. Plant Biol. 2013; 64:807-838. [PubMed: 23373698] 
15. Caporaso JG, Kuczynski J, Stombaugh J, Bittinger K, Bushman FD, Costello EK, et al. QIIME allows analysis of high-throughput community sequencing data. Nat. Methods. 2010; 7:335-336. [PubMed: 20383131]

16. Castro HF, Classen AT, Austin EE, Norby RJ, Schadt CW. Soil Microbial Community Responses to Multiple Experimental Climate Change Drivers. Appl. Environ. Microb. 2009; 76:999-1007.

17. Cleland EE, Chuine I, Menzel A, Mooney HA, Schwartz MD. Shifting plant phenology in response to global change. Trends Ecol. Evol. 2007; 22:357-365. [PubMed: 17478009]

18. Crimmins T, Crimmins M, Bertelsen C. Spring and summer patterns in flowering onset, duration, and constancy across a water-limited gradient. Am. J. Bot. 2013; 100:1137-1147. [PubMed: 23709634]

19. Edgar RC. UPARSE: highly accurate OTU sequences from microbial amplicon reads. Nat. Methods. 2013; 10:996-998. [PubMed: 23955772]

20. Ettema CH, Wardle DA. Spatial soil ecology. Trends Ecol. Evol. 2002; 17:177-183.

21. Fierer N, Jackson RB. The diversity and biogeography of soil bacterial communities. P. Natl. Acad. Sci. USA. 2006; 103:626-631.

22. Forrest J, Miller-Rushing AJ. Toward a synthetic understanding of the role of phenology in ecology and evolution. Philos. T. Roy. Soc. B-Biol. Sci. 2010; 365:3101-3112.

23. Ghalambor CK, McKay JK, Carroll SP, Reznick DN. Adaptive versus non-adaptive phenotypic plasticity and the potential for contemporary adaptation in new environments. Funct. Ecol. 2007; 21:394-407.

24. van der Heijden MGA, Bardgett RD, van Straalen NM. The unseen majority: soil microbes as drivers of plant diversity and productivity in terrestrial ecosystems. Ecol. Lett. 2008; 11:296-310. [PubMed: 18047587]

25. Hol WHG, de Boer W, Termorshuizen AJ, Meyer KM, Schneider JHM, van Dam NM, et al. Reduction of rare soil microbes modifies plant-herbivore interactions. Ecol. Lett. 2010; 13:292301. [PubMed: 20070364]

26. Kembel SW, Wu M, Eisen JA, Green JL. Incorporating 16S Gene Copy Number Information Improves Estimates of Microbial Diversity and Abundance. PLoS Comput. Biol. 2012; 8:e1002743. [PubMed: 23133348]

27. Korves T, Bergelson J. A developmental response to pathogen infection in Arabidopsis. Plant Physiol. 2003; 133:339-347. [PubMed: 12970499]

28. Lau JA, Lennon JT. Evolutionary ecology of plant-microbe interactions: soil microbial structure alters selection on plant traits. New Phytol. 2011; 192:215-224. [PubMed: 21658184]

29. Lau JA, Lennon JT. Rapid responses of soil microorganisms improve plant fitness in novel environments. P. Natl. Acad. Sci. USA. 2012; 109:14058-14062.

30. Lee C-R, Mitchell-Olds T. Complex trait divergence contributes to environmental niche differentiation in ecological speciation of Boechera stricta. Mol. Ecol. 2013; 22:2204-2217. [PubMed: 23432437]

31. Lundberg DS, Lebeis SL, Paredes SH, Yourstone S, Gehring J, Malfatti S, et al. Defining the core Arabidopsis thaliana root microbiome. Nature. 2012; 488:86-90. [PubMed: 22859206]

32. Lundberg DS, Yourstone S, Mieczkowski P, Jones CD, Dangl JL. Practical innovations for highthroughput amplicon sequencing. Nat. Methods. 2013; 10:999-1002. [PubMed: 23995388]

33. MacColl ADC. The ecological causes of evolution. Trends Ecol. Evol. 2011; 26:514-522. [PubMed: 21763030]

34. Magoč T, Salzberg SL. FLASH: fast length adjustment of short reads to improve genome assemblies. Bioinformatics. 2011; 27:2957-2963. [PubMed: 21903629]

35. Miner B, Sultan S, Morgan S, Padilla D, Relyea R. Ecological consequences of phenotypic plasticity. Trends Ecol. Evol. 2005; 20:685-692. [PubMed: 16701458]

36. Oksanen, J.; Blanchet, F.; Kindt, R.; Legendre, P.; Minchin, P.; O'Hara, RB., et al. vegan: Community Ecology Package. R package version 2.0-9.. 2013. http://CRAN.R-project.org/ package=vegan

37. Pigliucci M, Schlichting C, Whitton J. Reaction norms of Arabidopsis. 2. Response to stress and unordered environmental variation. Funct. Ecol. 1995; 9:537-547. 
38. R Core Team. R: A language and environment for statistical computing. R Foundation for Statistical Computing; Vienna, Austria: 2013. http://www.R-project.org/

39. Richards CL, Bossdorf O, Muth NZ, Gurevitch J, Pigliucci M. Jack of all trades, master of some? On the role of phenotypic plasticity in plant invasions. Ecol. Lett. 2006; 9:981-993. [PubMed: 16913942]

40. Rodríguez H, Fraga R. Phosphate solubilizing bacteria and their role in plant growth promotion. Biotechnol. Adv. 1999; 17:319-339. [PubMed: 14538133]

41. Rushworth CA, Song B-H, Lee C-R, Mitchell-Olds T. Boechera, a model system for ecological genomics. Mol. Ecol. 2011; 20:4843-4857. [PubMed: 22059452]

42. Ryser P, Sauder W. Effects of heavy-metal-contaminated soil on growth, phenology and biomass turnover of Hieracium piloselloides. Environ. Pollut. 2006; 140:52-61. [PubMed: 16185797]

43. Song BH, Clauss MJ, Pepper A, Mitchell-Olds T. Geographic patterns of microsatellite variation in Boechera stricta, a close relative of Arabidopsis. Mol. Ecol. 2006; 15:357-369. [PubMed: 16448406]

44. Stanton ML, Roy BA, Thiede DA. Evolution in stressful environments. I. Phenotypic variability, phenotypic selection, and response to selection in five distinct environmental stresses. Evolution. 2000; 54:93-111. [PubMed: 10937187]

45. Strauss, SY.; Whittall, JB. Non-pollinator agents of selection on floral traits.. In: Harder, LD.; Barrett, SCH., editors. Ecology and Evolution of Flowers. Oxford University Press, Inc.; New York, USA: 2006. p. 120-138.

46. Thompson JD. Phenotypic plasticity as a component of evolutionary change. Trends Ecol. Evol. 1991; 6:246-249. [PubMed: 21232470]

47. Vorholt JA. Microbial life in the phyllosphere. Nat. Rev. Microbiol. 2012; 10:828-840. [PubMed: 23154261]

48. Wade M, Kalisz S. The causes of natural selection. Evolution. 1990; 44:1947-1955.

49. Wickham, H. ggplot2: elegant graphics for data analysis. Springer; New York: 2009.

50. Van Zandt PA, Mopper S. Delayed and carryover effects of salinity on flowering in Iris hexagona (Iridaceae). Am. J. Bot. 2002; 89:1847-1851. [PubMed: 21665613] 


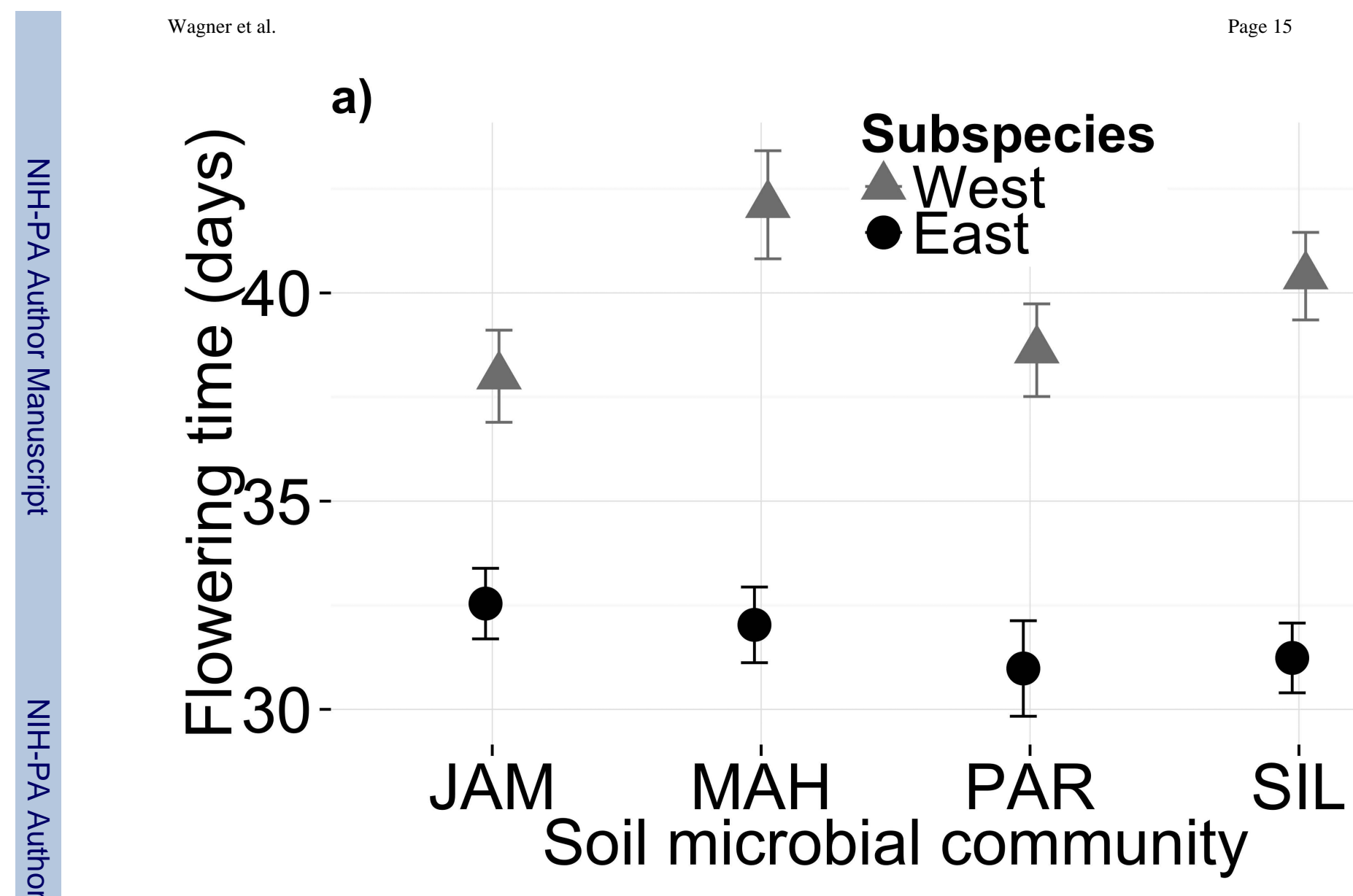




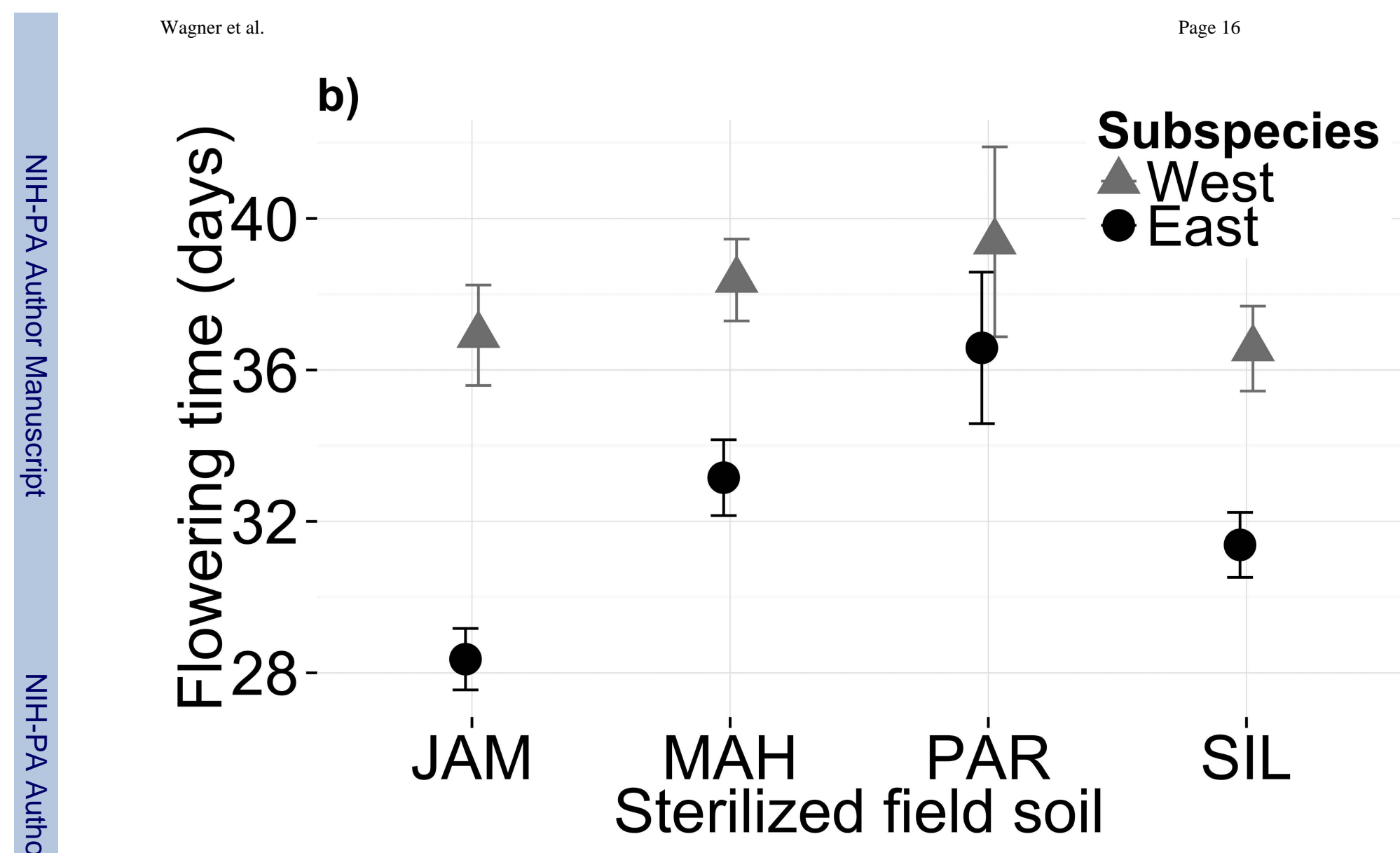




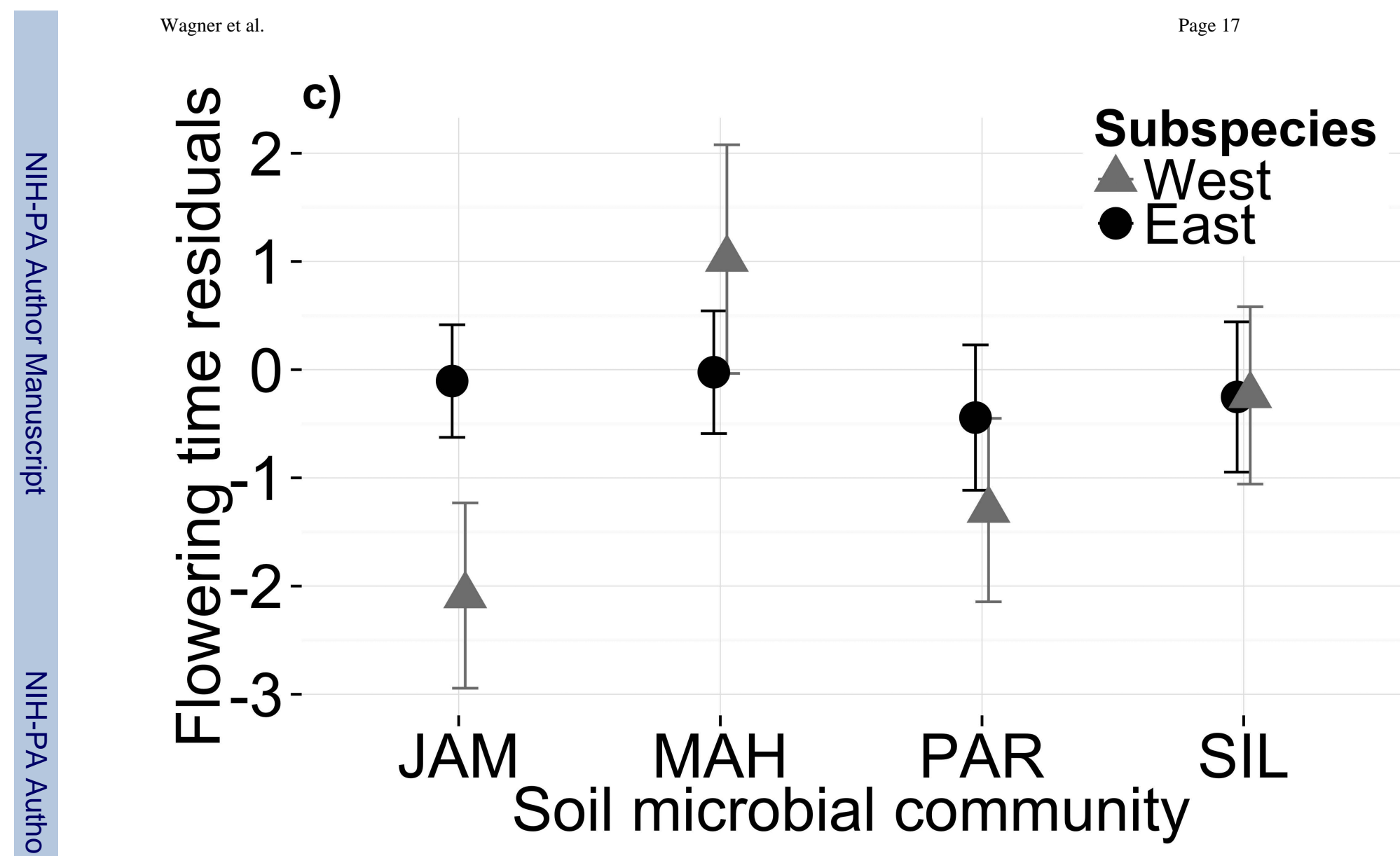




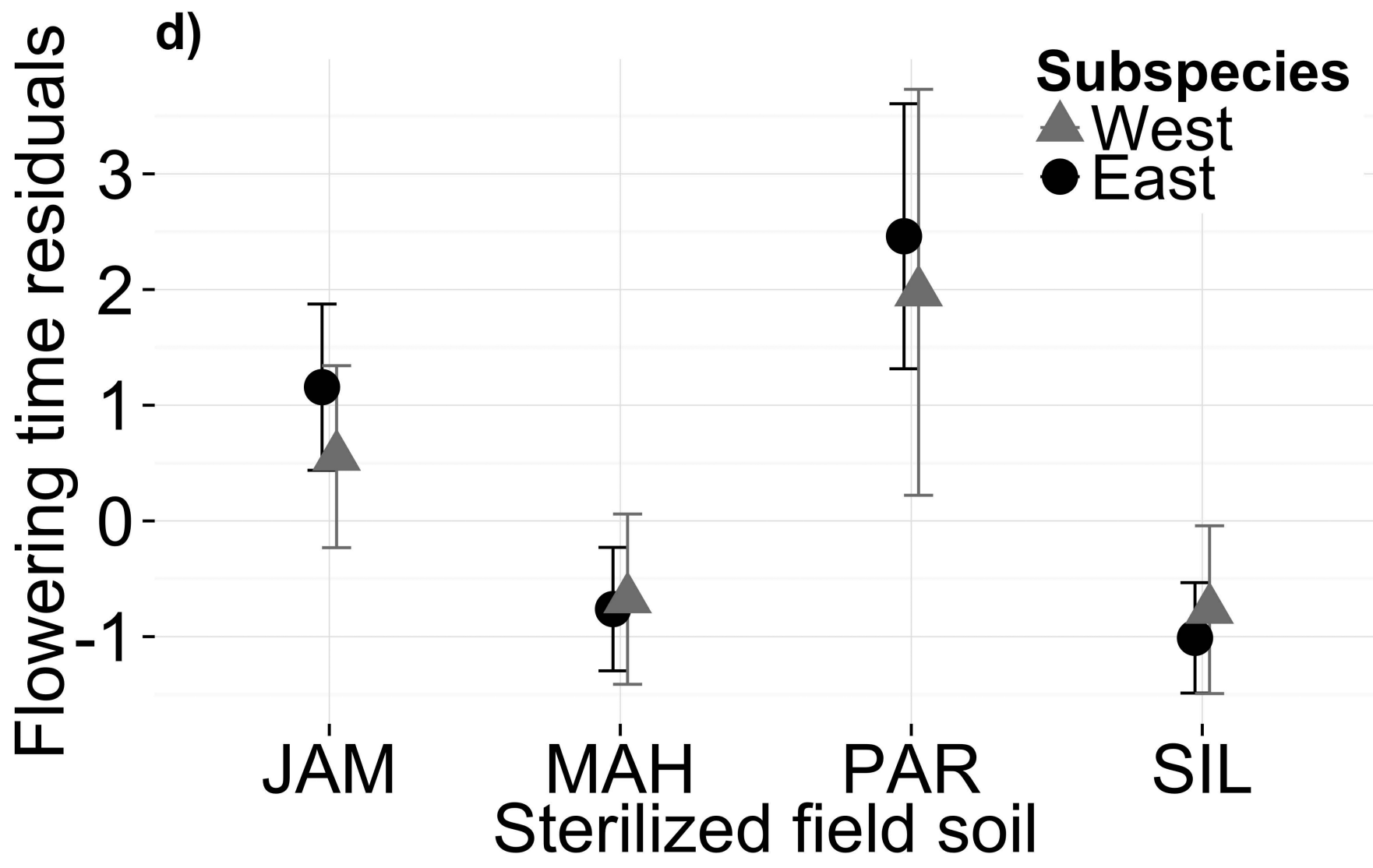

Figure 1.

Variation in both soil microbiota and soil chemistry alters flowering phenology. Mean flowering time post-vernalization (raw data, in days) is shown for each subspecies in (a) four microbial treatments and (b) four sterilized field soils. Panels (c) and (d) show mean flowering time residuals, after controlling for genetic differences and growth covariates, for each subspecies in biotic and abiotic treatments, respectively. Error bars depict one standard error from the mean. Parameter estimates and standard errors are reported in Table S3 
a)

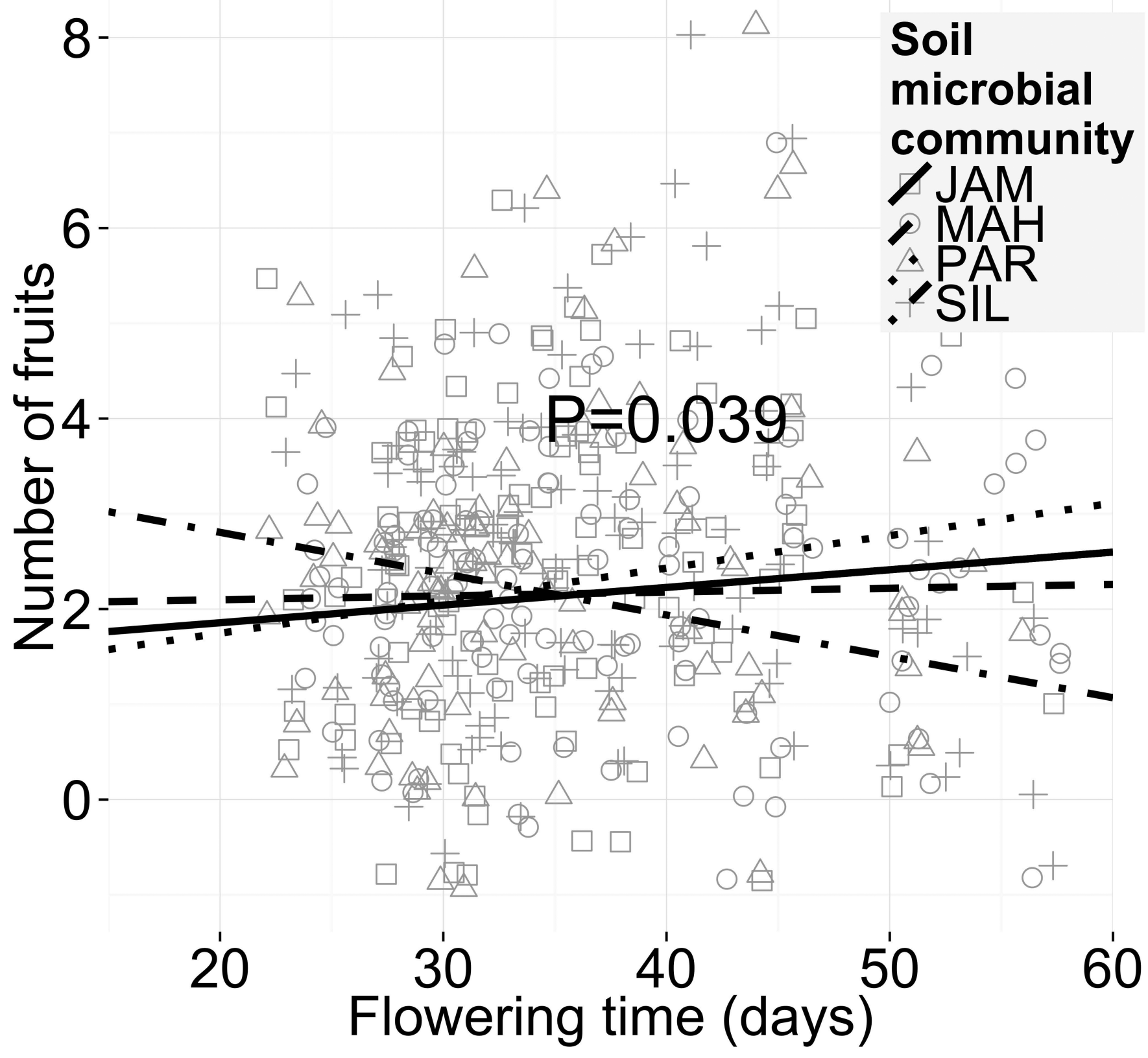


$12^{-b)}$

8 -

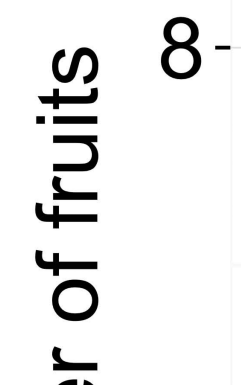

20

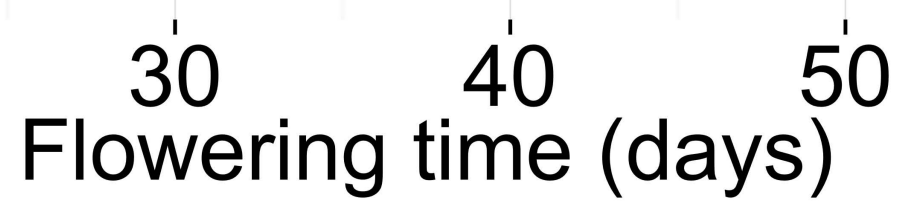

Sterilized field soil /JAM $\checkmark \mathrm{MAH}$ - PAR

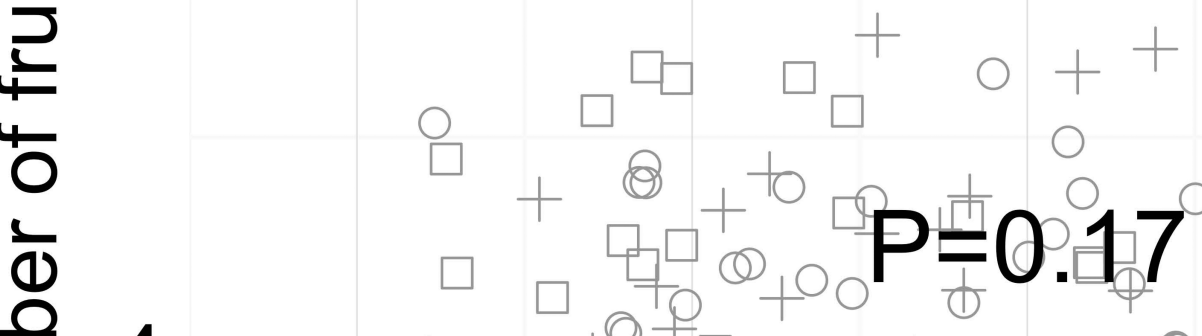

है

0

( )

to $\triangle$
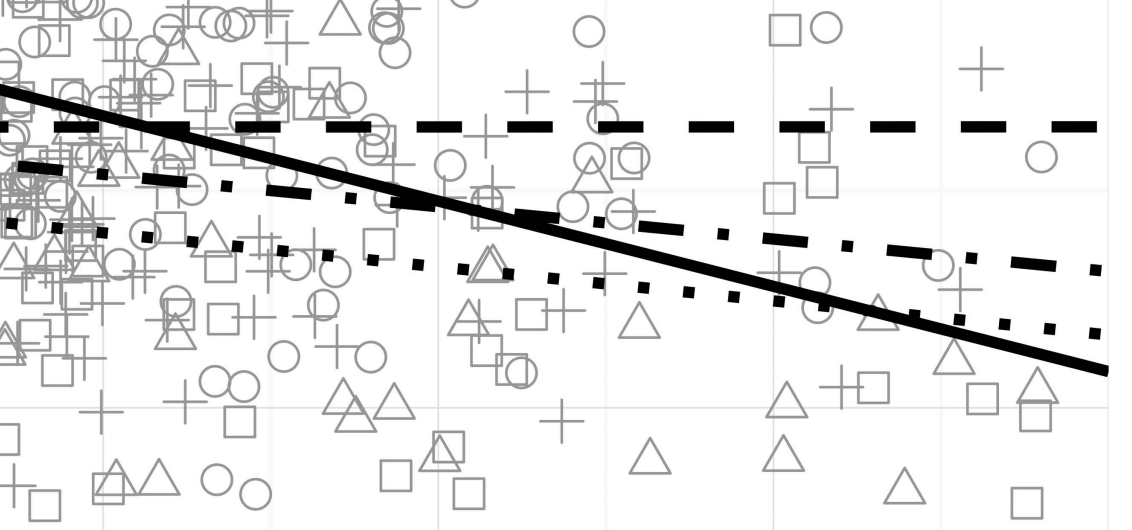
5.0-
c)

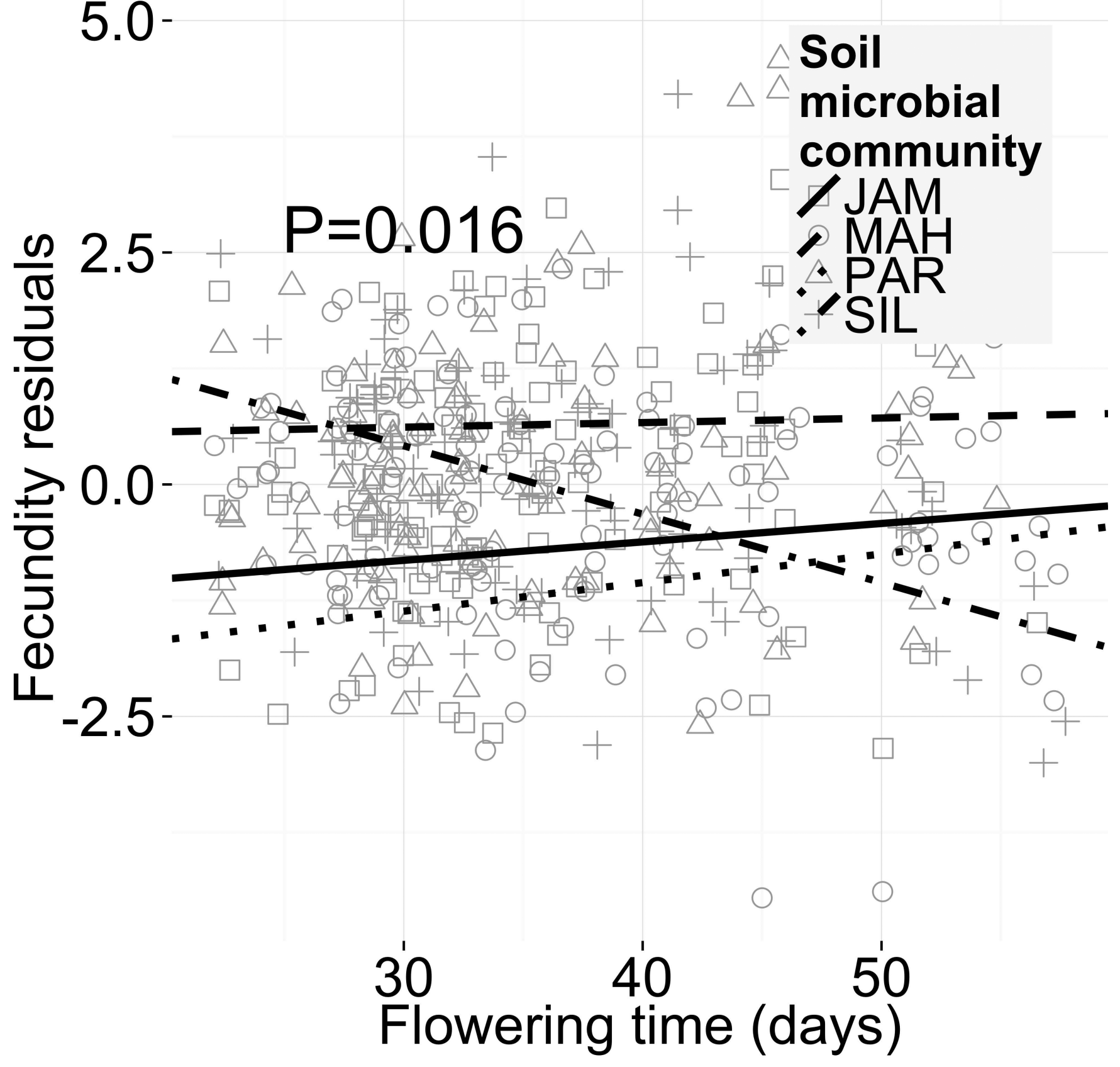


d)

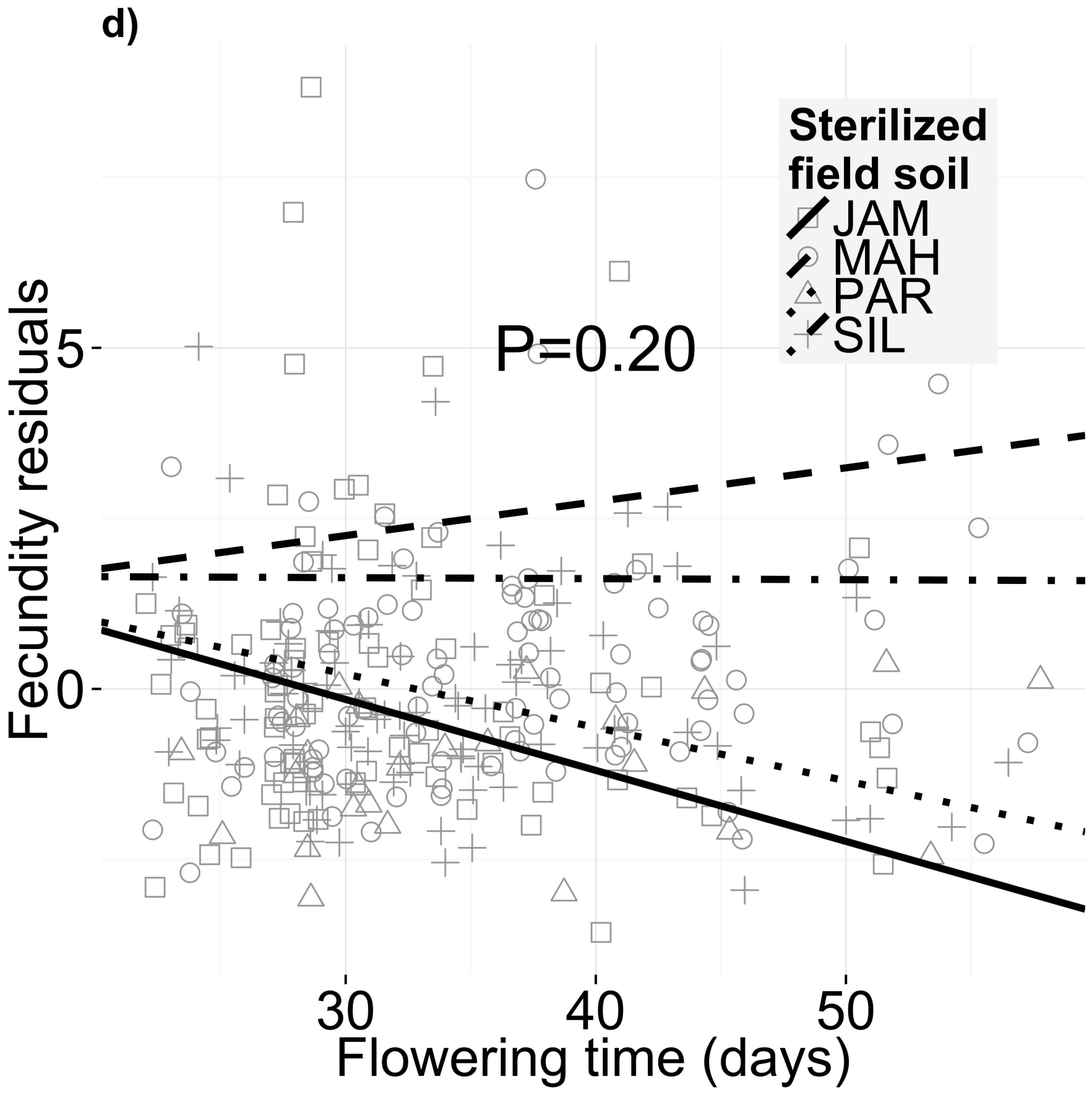

Figure 2.

Different soil microbiota, but not sterilized field soils, alter selection on flowering time.

Fecundity as a function of flowering time is shown for four different $(\mathrm{a}, \mathrm{c})$ soil microbial communities and (b,d) sterilized field soils. Panels (a) and (b) depict selection differentials on flowering phenology, i.e. both direct selection on flowering time and indirect selection on correlated traits, after controlling for block effects and genetic differences in fitness. The statistics for the selection differential model are in Table S5. Panels (c) and (d) depict selection gradients on flowering phenology, i.e. only direct selection on flowering time after 
controlling for selection on growth-related covariates in addition to block effects and genetic differences in fitness (statistics in Table 2) 


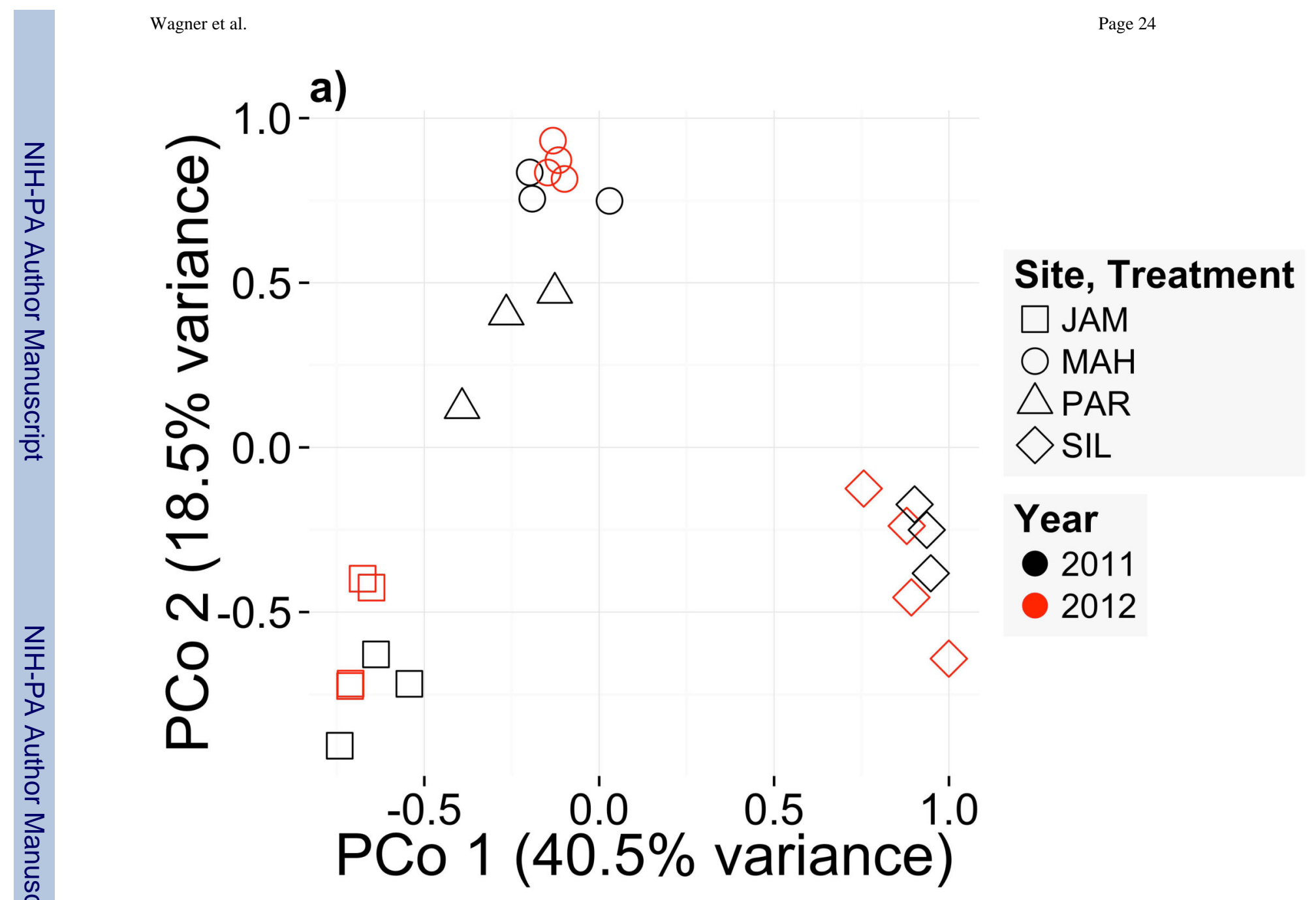


b)

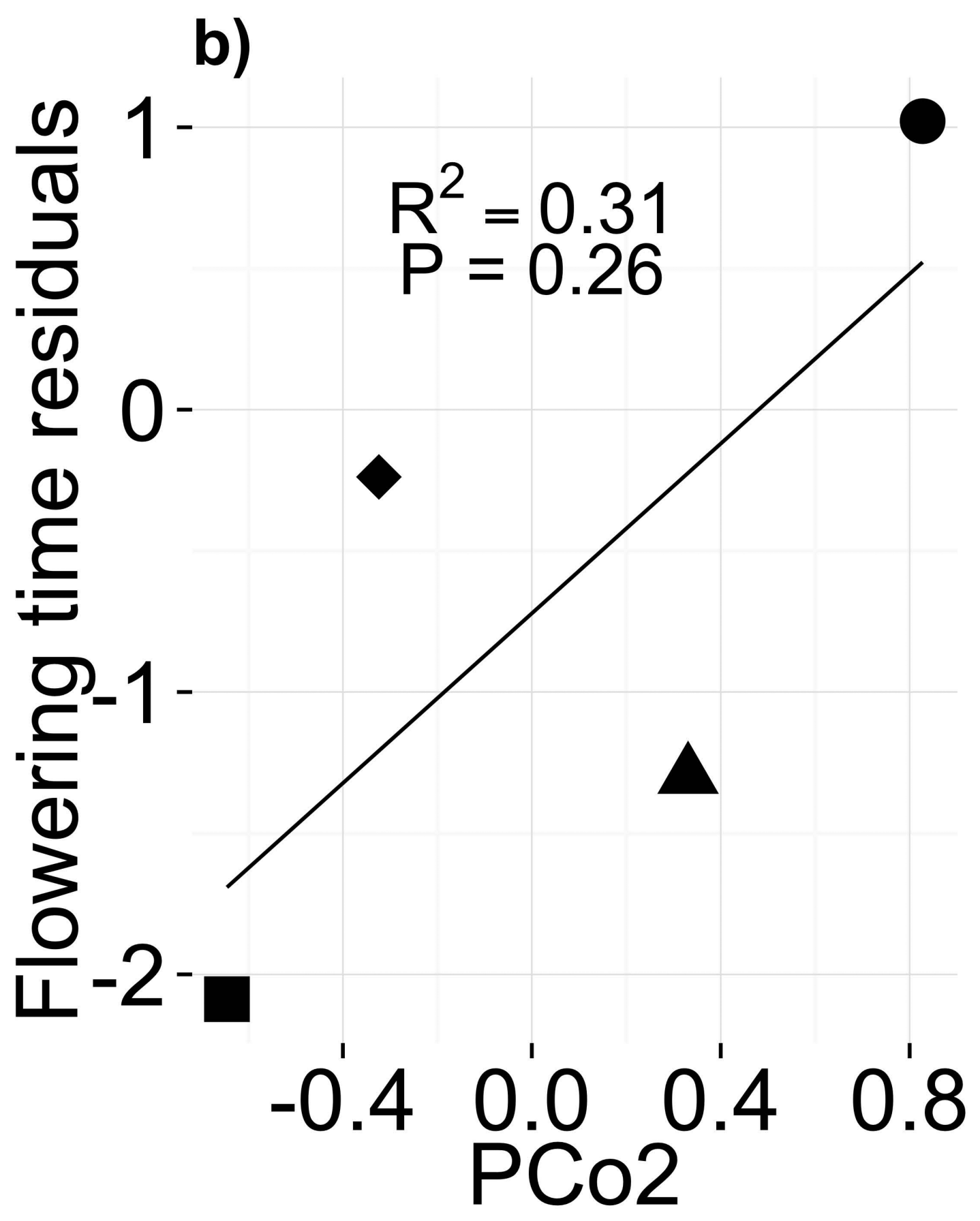




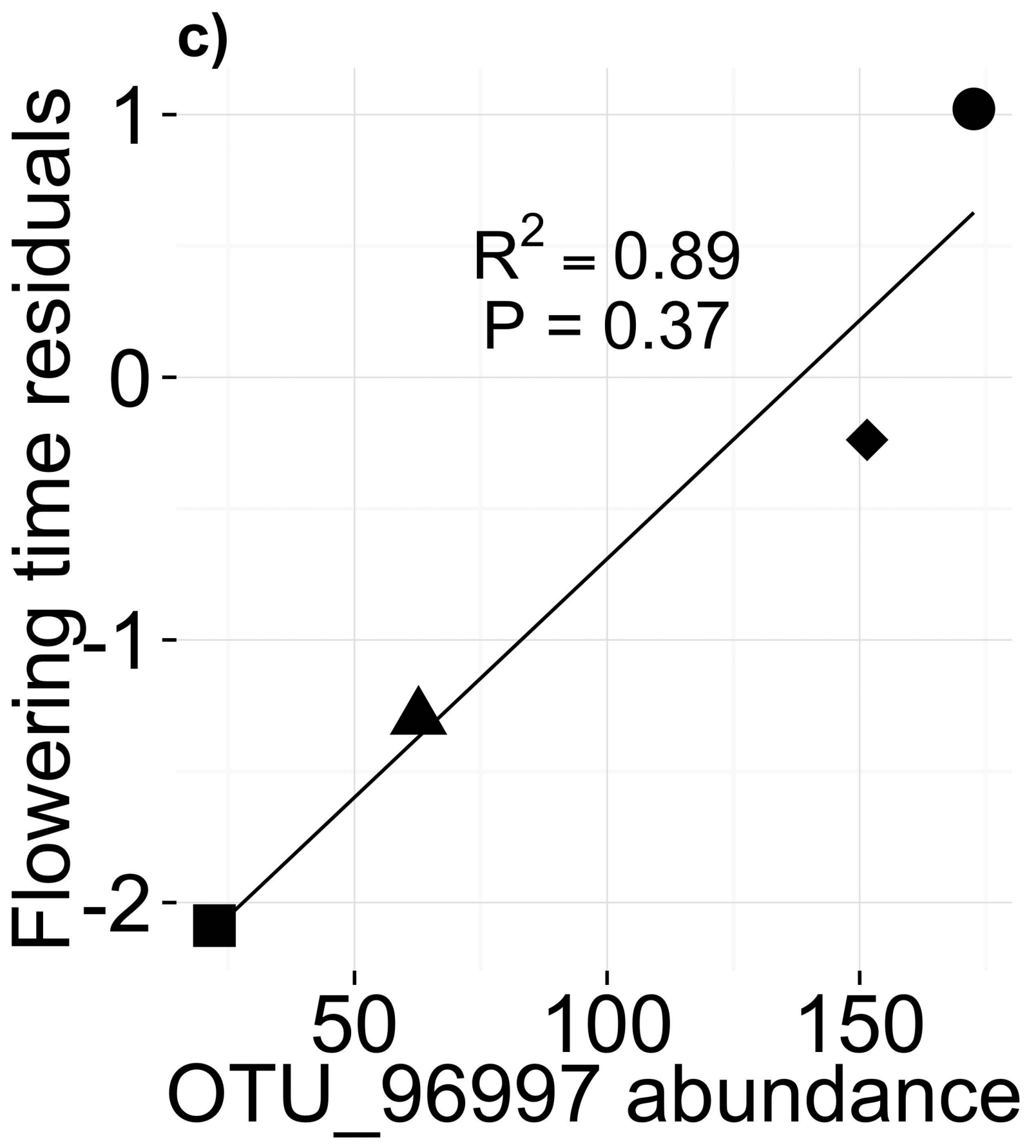

Figure 3.

(a) Ordination of Bray-Curtis dissimilarities of rarefied microbial abundances shows clustering of soil samples by field site of origin (ADONIS $P=0.003$ ), but not by collection year (ADONIS $P=0.56$ ). (b) Flowering time response of western genotypes (residuals, after controlling for block effects, genetic differences, and growth rate) to a gradient of soil microbiome $\mathrm{PCo} 2$, a candidate predictor of flowering time. (c) Flowering time response of 
western genotypes (residuals, as in [b]) to abundance of candidate microbe OTU_96997, chosen for its high loading onto PCo2 (Table S6; Appendix S3) 


\section{Table 1}

Statistics for REML mixed models of flowering time for (a) soil microbial community treatments and (b) sterilized field soil treatments. All effects are fixed except for Block, Genotype(Subspecies), and Genotype(Subsp.) $\times$ Treatment. For fixed effects the test statistic $F$ is reported. For random effects, the test statistic $\chi^{2}$ is reported, calculated as twice the difference between log likelihoods of the full model and the model with the random factor excluded. A similar model that did not control for growth-related covariates yielded similar results (Table S4)

\begin{tabular}{|c|c|c|c|c|c|c|}
\hline & \multicolumn{3}{|c|}{ (a) Soil microbial communities $\left(N=451\right.$, Adj. $\left.\mathrm{R}^{2}=0.74\right)$} & \multicolumn{3}{|c|}{ (b) Sterilized field soils $\left(N=336, \mathrm{Adj} . \mathbf{R}^{2}=0.72\right)$} \\
\hline & d.f. & $F$ or $\chi^{2}$ & $P$ & d.f. & $F$ or $\chi^{2}$ & $\boldsymbol{P}$ \\
\hline Treatment & 3,87 & 4.242 & $0.0076^{*}$ & 3,96 & 6.586 & 0.0004 \\
\hline Subspecies & 1,67 & 6.861 & 0.0109 & 1,64 & 0.085 & 0.7717 \\
\hline Treatment $\times$ Subspecies & 3,88 & 2.346 & 0.0782 & 3,91 & 0.206 & 0.8919 \\
\hline Genotype (Subspecies) & 2 & 30.08 & $<0.0001$ & 2 & 0.864 & $>0.05$ \\
\hline Geno.(Ssp.) $\times$ Treatment & 6 & 0.000 & $>0.05$ & 6 & 0.160 & $>0.05$ \\
\hline Block & 1 & 0.083 & $>0.05$ & 1 & 0.785 & $>0.05$ \\
\hline Elongation rate & 1,428 & 304.2 & $<0.001$ & 1,278 & 342.9 & $<0.0001$ \\
\hline Height at flowering & 1,387 & 239.0 & $<0.001$ & 1,240 & 278.1 & $<0.0001$ \\
\hline Leaves per mm stem & 1,428 & 15.96 & $<0.001$ & 1,298 & 21.38 & $<0.0001$ \\
\hline
\end{tabular}

Parameter estimates are listed in Table S4. Bold type indicates significance of at least $P<0.05$

* Permutational ANOVA confirmed significance at $P=0.005$ 


\section{Table 2}

Statistics for REML mixed models of reproductive fitness for (a) soil microbial community treatments and (b) sterilized field soil treatments. All effects are fixed except for Block, Genotype(Subspecies), and Genotype(Subsp.) $\times$ Treatment. For fixed effects the test statistic $F$ is reported. For random effects, the reported test statistic is $\chi^{2}$, calculated as twice the difference between log likelihoods of the full model and the model with the random factor excluded. Note that in this model "flowering time" reflects the selection gradient, i.e. direct selection on flowering time after controlling for selection on growth-related covariates. A similar model without growth covariates revealed that the selection differential, i.e. a measure of combined direct and indirect selection on flowering time, behaves similarly with respect to the flowering time $x$ treatment interaction (Table S5)

\begin{tabular}{|c|c|c|c|c|c|c|}
\hline & \multicolumn{3}{|c|}{ (a) Soil microbial communities $\left(N=439\right.$, Adj. $\left.R^{2}=0.26\right)$} & \multicolumn{3}{|c|}{ (b) Sterilized field soils ( $N=331$, Adj. $R^{2}=0.47$} \\
\hline & d.f. & $F$ or $\chi^{2}$ & $\boldsymbol{P}$ & d.f. & $F$ or $\chi^{2}$ & $P$ \\
\hline Treatment & 3,86 & 2.123 & 0.1032 & 3,110 & 5.849 & 0.0010 \\
\hline Subspecies & 1,69 & 7.351 & 0.0085 & 1,64 & 16.13 & 0.0002 \\
\hline Flowering time & 1,403 & 0.009 & 0.9240 & 1,308 & 0.376 & 0.5399 \\
\hline Treatment $\times$ Subspecies & 3,97 & 2.053 & 0.1115 & 3,109 & 1.170 & 0.3247 \\
\hline Treatment $\times$ Fl. time & 3,246 & 3.489 & $0.0164^{*}$ & 3,305 & 1.552 & 0.2011 \\
\hline Genotype (Subspecies) & 2 & 13.79 & $<0.01$ & 2 & 0.145 & $>0.05$ \\
\hline Geno.(Ssp. $) \times$ Treatment & 6 & 0.0 & $>0.05$ & 6 & 4.081 & $>0.05$ \\
\hline Block & 1 & 0.0008 & $>0.05$ & 1 & 0.130 & $>0.05$ \\
\hline Elongation rate & 1,376 & 0.0780 & 0.7801 & 1,300 & 0.1829 & 0.6692 \\
\hline Height at flowering & 1,385 & 3.116 & 0.0783 & 1,298 & 0.1291 & 0.7196 \\
\hline Leaves per $\mathrm{mm}$ stem & 1,381 & 15.03 & 0.0001 & 1,303 & 0.1157 & 0.7339 \\
\hline
\end{tabular}

Parameter estimates are listed in Table S3. Bold type indicates significance of at least $P<0.05$

* Permutational ANOVA confirmed significance at $P=0.024$ 


\section{Table 3}

(a) Mean flowering time of western genotypes (residuals after controlling for genetic differences and growth rates), mean PCo2 score, and mean abundance of OTU 96997 in each microbial community. Flowering time data come from the greenhouse experiment, community composition data come from soil samples taken from the same field sites as the inocula for the greenhouse experiment. This set of data can be informative for finding subsets of the microbial community associated with change in flowering time (Fig. 3; Appendix S3). (b) Copy number-adjusted relative abundances of dominant phyla in slow-flowering MAH and late-flowering JAM soils. (c) Copy number-adjusted relative abundances in the full dataset ("All OTUs") and the subset of $5 \%$ of OTUs most strongly correlated with PCo2, a putative predictor of flowering time. Significance of enrichment/depletion was determined by Wilcoxon Rank Sum tests and adjusted using the BenjaminiHochberg false discovery rate

\begin{tabular}{|c|c|c|c|c|c|c|c|c|}
\hline (a) Site & \multicolumn{3}{|c|}{ Flowering time (residual) } & \multicolumn{3}{|c|}{ PCo2 } & \multicolumn{2}{|c|}{ OTU 96997 (individuals, \pm 1 standard error) } \\
\hline JAM & \multicolumn{3}{|c|}{$-2.088 \pm 0.856$} & \multicolumn{3}{|c|}{$-0.646 \pm 0.068$} & \multicolumn{2}{|c|}{$22.27 \pm 4.04$} \\
\hline MAH & \multicolumn{3}{|c|}{$1.022 \pm 1.057$} & \multicolumn{3}{|c|}{$0.827 \pm 0.024$} & \multicolumn{2}{|c|}{$172.60 \pm 16.67$} \\
\hline PAR & \multicolumn{3}{|c|}{$-1.298 \pm 0.848$} & \multicolumn{3}{|c|}{$0.331 \pm 0.107$} & \multicolumn{2}{|c|}{$62.67 \pm 12.90$} \\
\hline SIL & \multicolumn{3}{|c|}{$-0.238 \pm 0.819$} & \multicolumn{3}{|c|}{$-0.324 \pm 0.068$} & \multicolumn{2}{|c|}{$151.47 \pm 49.94$} \\
\hline \multicolumn{2}{|l|}{ Phylum } & (b) JAM & \multicolumn{2}{|c|}{ МАН } & $P_{F D R}$ & (c) All & PCo2 & $P_{F D R}$ \\
\hline \multicolumn{2}{|c|}{ Proteobacteria } & $17.3 \%$ & \multicolumn{2}{|c|}{$26.7 \%$} & 0.002 & $24.8 \%$ & $23.5 \%$ & 0.49 \\
\hline \multicolumn{2}{|c|}{ Acidobacteria } & $21.3 \%$ & \multicolumn{2}{|c|}{$15.3 \%$} & 0.002 & $17.6 \%$ & $19.0 \%$ & 0.40 \\
\hline \multicolumn{2}{|c|}{ Verrucomicrobia } & $19.3 \%$ & \multicolumn{2}{|c|}{$15.3 \%$} & 0.054 & $17.0 \%$ & $22.5 \%$ & 0.004 \\
\hline \multicolumn{2}{|c|}{ Actinobacteria } & $16.0 \%$ & \multicolumn{2}{|c|}{$17.8 \%$} & 0.21 & $16.0 \%$ & $14.3 \%$ & 0.036 \\
\hline \multicolumn{2}{|c|}{ Gemmatimonadetes } & $9.5 \%$ & \multicolumn{2}{|c|}{$9.4 \%$} & 1 & $7.7 \%$ & $9.6 \%$ & 0.036 \\
\hline \multicolumn{2}{|c|}{ Chloroflexi } & $5.7 \%$ & \multicolumn{2}{|c|}{$7.3 \%$} & 0.054 & $6.0 \%$ & $5.5 \%$ & 0.40 \\
\hline \multicolumn{2}{|c|}{ Planctomycetes } & $3.4 \%$ & & & 0.054 & $4.0 \%$ & $0.43 \%$ & $<0.001$ \\
\hline Bactero & etes & $2.9 \%$ & & & 1 & $3.7 \%$ & $2.4 \%$ & 0.012 \\
\hline Crenarc & aeota & $0.06 \%$ & & $5 \%$ & 0.002 & $0.70 \%$ & $0.95 \%$ & 0.61 \\
\hline
\end{tabular}

